\title{
The effect of local cryotherapy on subjective and objective recovery characteristics following an exhaustive jump protocol
}

\author{
This article was published in the following Dove Press journal: \\ Open Access Journal of Sports Medicine \\ 22 August 2016 \\ Number of times this article has been viewed
}

\section{Erich Hohenauer ${ }^{1-3}$ \\ Peter Clarys ${ }^{3}$ \\ Jean-Pierre Baeyens ${ }^{2-4}$ \\ Ron Clijsen ${ }^{1-3}$}

'Department of Business Economics, Health and Social Care, University of Applied Sciences and Arts of Southern Switzerland, Landquart, Switzerland; ${ }^{2}$ University College Physiotherapy, Thim van der Laan, Landquart, Switzerland; ${ }^{3}$ Faculty of Physical Education and Physiotherapy, Vrije Universiteit Brussel, Brussels, Belgium; ${ }^{4} \mathrm{Faculty}$ of Medicine and Health Sciences, University of Antwerp, Antwerp, Belgium
Correspondence: Erich Hohenauer Department of Business Economics, Health and Social Care, University of Applied Sciences and Arts of Southern Switzerland, Weststrasse 8, CH-7302 Landquart, Switzerland Tel $+4|8| 3000175$

Fax +4I 8I 300 01 71

Email erich.hohenauer@supsi.ch

\begin{abstract}
The purpose of this controlled trial was to investigate the effects of a single local cryotherapy session on the recovery characteristics over a period of 72 hours. Twentytwo young and healthy female $(n=17$; mean age: $21.9 \pm 1.1$ years) and male $(n=5 ;$ mean age: $25.4 \pm 2.8$ years $)$ adults participated in this study. Following an exhaustive jump protocol $(3 \times 30$ countermovement jumps), half of the participants received either a single local cryotherapy application $\left(+8^{\circ} \mathrm{C}\right)$ or a single local thermoneutral application $\left(+32^{\circ} \mathrm{C}\right)$ of 20 -minute duration using two thigh cuffs. Subjective measures of recovery (delayed-onset muscle soreness and ratings of perceived exertion) and objective measures of recovery (vertical jump performance and peak power output) were assessed immediately following the postexercise applications ( 0 hours) and at 24 hours, 48 hours, and 72 hours after the jump protocol. Local cryotherapy failed to significantly affect any subjective recovery variable during the 72-hour recovery period $(P>0.05)$. After 72 hours, the ratings of perceived exertion were significantly lower in the thermoneutral group compared to that in the cryotherapy group $(P=0.002)$. No significant differences were observed between the cryotherapy and the thermoneutral groups with respect to any of the objective recovery variables. In this experimental study, a 20-minute cryotherapy cuff application failed to demonstrate a positive effect on any objective measures of recovery. The effects of local thermoneutral application on subjective recovery characteristics were superior when compared to the effects of local cryotherapy application at 72 hours postapplication.
\end{abstract}

Keywords: cryotherapy, cold cuff, muscle damage, recovery

\section{Introduction}

The use of cryotherapy as a postexercise recovery modality has been increasing in popularity. ${ }^{1,2}$ The effects of cryotherapy are believed to be a result of peripheral vasoconstriction, which leads to the reduction of the inflammatory process due to decreased cell metabolism. ${ }^{3,4}$ Commonly used cryotherapy procedures consist of external applications, such as cold packs, ${ }^{5}$ ice cuffs,${ }^{6}$ cold air, ${ }^{7}$ ice sprays,${ }^{8}$ cold-water immersion (CWI), ${ }^{9}$ whole-body cryotherapy chambers, ${ }^{10}$ whole-body cryochambers using liquid nitrogen, ${ }^{11}$ cooling vests, ${ }^{12}$ or any other combination of these methods, as well as cold-water ingestion. ${ }^{13,14}$ The latter procedure has resulted in conflicting results. $^{15,16}$ 
However, researchers have presented conflicting data on the use of localized cryotherapy application. King and Duffield ${ }^{17}$ reported no significant postrecovery vertical jump performance (VJP) differences, as well as significantly lower ratings on both subjective measures, when cryotherapy and active recovery procedures were utilized. Tseng et $\mathrm{al}^{5}$ presented opposing data, stating that cryotherapy had no influence on postrecovery performance (objective measure), with significantly higher reported ratings of perceived exertion (RPE). A recent meta-analysis by Hohenauer et al ${ }^{1}$ involving cryotherapy use after exhaustive exercise reported significantly lowered ratings for both subjective measures for up to 96 hours, compared to other recovery strategies.

Validated methods for evaluating cryotherapy efficacy have included subjective assessments (delayed-onset muscle soreness $[\mathrm{DOMS}]^{18}$ and RPE), ${ }^{19}$ while the countermovement jump (CMJ) performance is an often-used objective measure. ${ }^{17,20}$ Symptoms of DOMS generally commence 12-24 hours after exercise-induced muscle damage (EIMD), which include tenderness on palpation and muscle stiffness during movement. ${ }^{21}$ One of the most common protocols for potentially preventing symptoms of DOMS is CWI. ${ }^{22}$ The philosophy of CWI follows the principles of cryotherapy listed herein. Although the use of CWI is very popular, its use is not always applicable in the field. Cold-water tubs, in which athletes can be immersed, are neither portable nor readily available for use. Furthermore, the water must be maintained at a specific temperature and requires constant monitoring. The risk of temperature fluctuation increases, which can lead to distortion of the results. Mobile and affordable instruments, which allow cooling at a constant temperature under field conditions, need to be scientifically evaluated.

As presented herein, there is no evidence in the literature that local cryotherapy has a positive effect on muscle recovery and/or physical performance. ${ }^{23}$ Therefore, the purpose of this study was to investigate the effects of a single local cryotherapy application over a 72-hour period after exhaustive exercise recovery using subjective and objective measures. We hypothesized that local cryotherapy would have a positive effect on recovery and lead to higher VJP (objective measure), as well as associated positive results on subjective, namely, DOMS and RPE, measures.

\section{Methods}

\section{Participants}

The sample size for both cold and thermoneutral conditions was determined in $\mathrm{G}^{*}$ Power (version 3.1.9.2; Franz Faul, University of Kiel, Kiel, Germany). The following design specifications were taken into account: $\alpha=0.05 ;(1-\beta)=0.8$; effect size $f=0.4$; test family $=F$-test; and statistical test $=$ repeated-measures analysis of variance (ANOVA), withinbetween interaction. The sample size estimated according to these specifications was 12 participants (actual power $=0.88$ ). Twenty-two healthy participants ( 17 females and five males), with mean age of 22.6 \pm 2 years (range: 20.1-29.8 years), regularly involved in moderate physical endurance activity, volunteered for this study. Eight females and three males were randomly assigned to the cold group (mean age: 22.8 \pm 2.3 years; range: $20.4-29$ years) and the remaining eleven (nine females and two males) were assigned to the thermoneutral group (mean age: $22.4 \pm 1.8$ years; range: 20-26.6 years). Mixed sex populations have already been used in the field of cryotherapy studies. ${ }^{24,25}$ Twenty-four participants wanted to take part in this study. Two participants had to be excluded due to current pain symptoms. The anthropometric characteristics of the participants can be observed in Table 1.

All contacted volunteer participants were screened to establish whether they meet the study criteria (Table 2). This

Table I Anthropometric data of the participants

\begin{tabular}{|c|c|c|c|}
\hline \multirow[t]{2}{*}{ Parameters } & \multirow[t]{2}{*}{ Total $(n=22)$} & \multicolumn{2}{|l|}{ Groups } \\
\hline & & $\begin{array}{l}\text { Cold } \\
\text { treatment } \\
(n=I I)\end{array}$ & $\begin{array}{l}\text { Thermoneutral } \\
\text { treatment } \\
(n=I I)\end{array}$ \\
\hline Height (cm) & $\begin{array}{l}170.3 \pm 7.5 \\
(159-184.6)\end{array}$ & $\begin{array}{l}172.5 \pm 8.3 \\
(159-184.6)\end{array}$ & $\begin{array}{l}168.1 \pm 6.2 \\
(160.8-184.2)\end{array}$ \\
\hline Weight (kg) & $\begin{array}{l}66.0 \pm 10.7 \\
(51.6-101.8)\end{array}$ & $\begin{array}{l}68.2 \pm 13.4 \\
51.6-101.8)\end{array}$ & $\begin{array}{l}63.7 \pm 7.1 \\
(54.4-77.6)\end{array}$ \\
\hline ELBF\% & $\begin{array}{l}28.4 \pm 8.0 \\
(14-39)\end{array}$ & $\begin{array}{l}27.8 \pm 9.0 \\
(14-39)\end{array}$ & $\begin{array}{l}29.0 \pm 7.2 \\
(14-36)\end{array}$ \\
\hline BMI & $\begin{array}{l}22.6 \pm 2.2 \\
20.1-29.8\end{array}$ & $\begin{array}{l}22.7 \pm 2.6 \\
(20.1-29.8)\end{array}$ & $\begin{array}{l}22.5 \pm 1.9 \\
(20.1-26.4)\end{array}$ \\
\hline
\end{tabular}

Note: Values are presented as mean \pm SD (range).

Abbreviations: BMI: body mass index; ELBF\%: estimated lower body fat, in percentage; SD, standard deviation.

Table 2 Detailed inclusion and exclusion criteria for this study

\begin{tabular}{ll}
\hline Inclusion criteria & Exclusion criteria \\
\hline Participants aged between & Allergy to cold (eg, Raynaud's \\
I 8 years and 30 years & disease) \\
Moderate physical activity & Cardiovascular diseases \\
(minimum: two times per week & Cardiac pacemaker and cardiac \\
endurance exercise, total time & arrhythmia \\
minimum: 2 hours; maximum: & Intake of any medication \\
3 times per week endurance exercise, & Pregnancy \\
total time maximum: 3 hours) & Skeletal deviations \\
No musculoskeletal surgery & Alcohol consumption during \\
No injuries at the torso or lower & 72-hour recovery \\
extremities in the previous 12 months & Recreational training during \\
No current pain symptoms & 72-hour recovery \\
\hline
\end{tabular}


study was approved by the Swiss Cantonal Ethical Committee of Zurich, KEK-ZH No 2015-0113. All participants provided written informed consent.

\section{Experimental design}

Testing was completed over a period of four consecutive days. To ensure exact follow-up measurements, participants were scheduled to complete the testing protocol at the same time each day. Baseline measurements were taken after participants were familiarized with the protocol. Familiarization with the CMJ was performed 1 week prior to the experiment. Participants were instructed to perform five maximum CMJs. ${ }^{26}$ One week after the familiarization session, the participants returned for the first of the four consecutive testing days.

\section{Subjective assessments}

The subjective recovery characteristics - DOMS (of the quadriceps muscle of both legs) and RPE - were rated on a $0-10 \mathrm{~cm}$ visual analog scale and on a 6-20 Borg scale, respectively. ${ }^{5,27}$ DOMS was always assessed in a squat position $\left(90^{\circ}\right.$ knee angle, with brief holding of maximum 3 seconds) ranging from zero (no soreness) to ten (severe soreness), while BORG ratings were always assessed in a standing position. ${ }^{27-29}$

DOMS and RPE were assessed directly after the recovery modality ( 0 hours) and always before the $\mathrm{CMJ}$ measurements during the whole experimental procedure (baseline, 0 hours, 24 hours, 48 hours, and 72 hours).

\section{Objective assessments}

Furthermore, baseline measurements included the assessment of the VJP using CMJ. The participants had to perform three maximal CMJs, ${ }^{26}$ with a 30 -second pause between the three jumps. The highest jump was recorded, and the peak power output (PPO, presented in watts) was calculated according to the formula used in the study of Sayers et al. ${ }^{30}$ The CMJ was performed on the Just Jump system (Probotics Inc, Huntsville, AL, USA), and the VJP was assessed as proposed earlier. ${ }^{31}$ VJP and PPO were assessed directly after the recovery modality ( 0 hours) and always after the subjective assessments during the whole experimental procedure (baseline, 0 hours, 24 hours, 48 hours, and 72 hours). CMJs show a high test-retest reliability (intraclass correlation coefficient [ICC] between 0.48 and 0.88 ) and validity 32,33

Directly after all baseline measurements were completed, the participants performed the exhaustive CMJ protocol. Approximately 30 seconds after the exercise protocol, the participants received the randomly assigned single cold or the single thermoneutral recovery modality. Approximately 30 seconds after receiving the recovery modality ( 0 hours), subjective recovery characteristics (DOMS and RPE) and objective recovery characteristics were assessed (VJP) and calculated (PPO). Follow-up measurements took place at exactly 24 hours, 48 hours, and 72 hours after the exercise protocol. The schematic representation of the test protocol is presented in Figure 1.

\section{Exhaustive CMJ protocol}

The participants did not perform any warm-up exercises or stretching prior to the jump protocol. The participants were instructed to perform maximally explosive $\mathrm{CMJ}(3 \times 30$ times or until exhaustion). Two researchers visually inspected the quality of the jumps (maximum and touching the ground with the fingers after each jump), corrected the participants if necessary, and verbally encouraged the participants. Previous studies showed that jump protocols, consisting of 100 jumps, induce local muscle damage. ${ }^{34,35}$ The participants were instructed to touch the ground with the fingers of both hands after each jump. Between each set, the participants had a 30 -second pause and were allowed to sit down. The 30 -second pause between the sets ensured accumulative overload of all participants. One female participant in the cold group had to stop in the third set after 15 repetitions due to her state of exhaustion. However, this female participant was not excluded from the analysis. The remaining 21 participants were able to complete the exhaustive jump protocol.

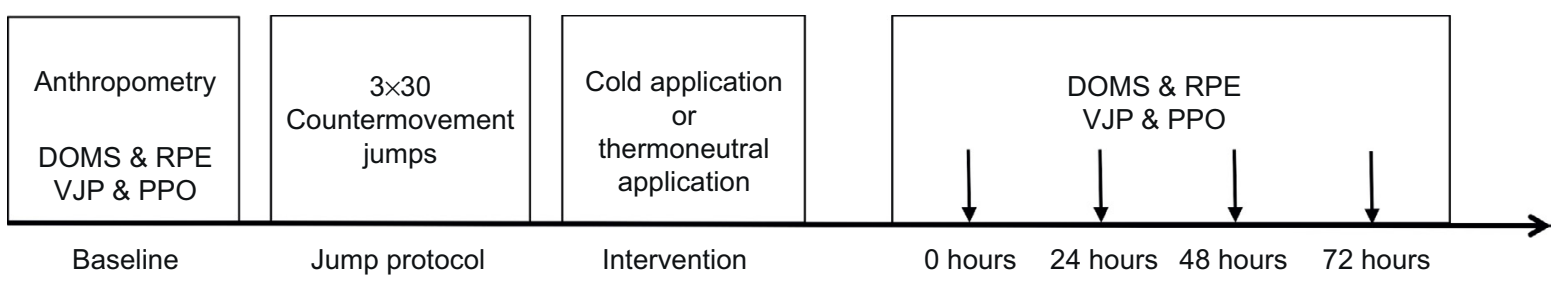

Figure I Schematic representation of the test protocol.

Note: Downward pointing arrows denote the time points when recovery characteristics were measured.

Abbreviations: DOMS, delayed-onset muscle soreness; PPO, peak power output; RPE, ratings of perceived exertion; VJP, vertical jump performance. 


\section{Recovery methods}

The recovery interventions (cold or thermoneutral) took place next to the exercise area and were applied using the Zamar ${ }^{\circledR}$ ZT Clinic from Zamar ${ }^{\circledR}$ Medical D.O.O. (Porec, Croatia, www.zamarmedical.com). The recovery modality was applied in a supine position for 20 minutes. A thigh cuff (Zamar ${ }^{\circledR}$ thigh cuff) was applied directly onto the skin and wrapped around each thigh, to ensure full contact between the inside of the cuff and the skin of the frontal thigh (from the basis patellae to the groin). Full contact between the cuff and the skin was provided when the inside area of the cuff had full contact with the covered skin of the thigh. The cuffs were wrapped around both thighs with a minimum level of pressure to avoid any compression effects. A minimum level of pressure was used only to ensure perfect alignment between the inside area of the cuff and the skin of the thigh. The Zamar ${ }^{\circledR}$ ZT Clinic ensured a constant temperature during the 20-minute application time in both cuffs. The Zamar therapy machine creates temperature changes by constantly circulating a lubrication mixture (consisting of propylene glycol and demineralized water) between the unit and the sleeve via hoses. The heat capacity of this lubrication mixture is slightly lower than that of normal water. The cold application temperature was set at $8^{\circ} \mathrm{C}$, whereas the temperature of the thermoneutral application was set at $32^{\circ} \mathrm{C}$. It has been demonstrated in a recently published review that water temperatures of $\sim 35^{\circ} \mathrm{C}$ are considered to be thermoneutral. ${ }^{36}$ The authors of this review pointed out that thermoneutral water temperatures have a range in the literature from $24^{\circ} \mathrm{C}$ to $<36^{\circ} \mathrm{C} .{ }^{20,37-43} \mathrm{To}$ minimize the possibility of bias, the participants were told not to report information about their temperature sensation to the researchers throughout the experiment.

\section{Statistical analysis}

Data analyses were carried out using the Statistical Package for Social Sciences for Windows (SPSS), version 19.0 (IBM Corporation, Armonk, NY, USA). Mauchly's test of sphericity was performed to test for homogeneity of differences of variance. Two-factor analysis for group (cold vs thermoneutral) and time (0 hours, 24 hours, 48 hours, and 72 hours) was conducted (repeated-measures ANOVA; multivariate ANOVA [MANOVA]). Group was used as the between-subjects factor and time as the within-subject factor. Bonferroni-corrected post hoc analyses were used to calculate the particular between-time differences. Effect sizes are expressed as partial eta ${ }^{2}\left(\eta_{\text {Partial }}^{2}\right)$ values of $0.1,0.3$, and $>0.5$, which were considered small, medium, and large, respectively. ${ }^{44}$ Pearson correlation coefficient was used to assess the potential linear relationships between the recovery variables. The level of significance was set at $P<0.05$. Descriptive statistics were used to describe the differences for DOMS and RPE (median \pm interquartile range) and for VJP and PPO (mean \pm standard deviation). PPO was calculated according to the preferred regression equation $(60.7 \times$ jump height $[\mathrm{cm}])$ $+(45.3 \times$ body weight $[\mathrm{kg}])-2,055=\mathrm{PPO}(\mathrm{W}){ }^{30}$

\section{Results Subjective measures of recovery}

A small but significant group effect was observed for DOMS $\left(F_{1,20}=5.411, P=0.03, \eta_{\text {Partial }}^{2}=0.213\right)$ but not for RPE $\left(F_{1,20}=3.070, P=0.09, \eta_{\text {Partial }}^{2}=0.133\right)$. However, no statistically significant post hoc differences between the groups were detected at any time point ( 0 hours, 24 hours, 48 hours, and 72 hours) for DOMS and RPE; only a trend with a small effect size for greater lowering of RPE in the thermoneutral group was observed after 72 hours $\left(P=0.06, \eta_{\text {Partial }}^{2}=0.162\right)$. The RPE values were significantly higher immediately after the exhaustive jump protocol ( 0 hours) in both groups $(P<0.05)$. However, these values returned to baseline after 24 hours in the thermoneutral group and remained at this level for up to 72 hours $(P=0.10$; no significant difference between baseline and 72-hour follow-up), compared to the results in the cold group ( $P=0.002$; significant difference between baseline and 72-hour follow-up). Furthermore, a medium significant increase over time was observed for DOMS $\left(F_{3,18}=6.988\right.$, $\left.P=0.003, \eta_{\text {Partial }}^{2}=0.538\right)$, while RPE significantly decreased over time (after 0 hours) with large effect sizes $\left(F_{3,18}=30.77\right.$, $\left.P<0.001, \eta_{\text {Partial }}^{2}=0.837\right)$. Significant post hoc differences were observed between specific points in time, for both DOMS and RPE (Figure 2). However, there was no significant group $\times$ time interaction, neither for DOMS $\left(F_{3,18}=0.203, P=0.89\right.$, $\left.\eta_{\text {Partial } 1}^{2}=0.033\right)$ nor for $\operatorname{RPE}\left(F_{3,18}=1.345, P=0.29, \eta_{\text {Partial }}^{2}=0.183\right)$.

\section{Objective measures of recovery}

No significant group effect was observed for $\operatorname{VJP}\left(F_{1,20}=0.917\right.$, $\left.P=0.35, \eta_{\text {Partial }}^{2}=0.044\right)$ and PPO $\left(F_{1,20}=0.002, P=0.96\right.$, $\eta_{\text {Partial }}^{2}<0.001$ ) during the whole recovery period (Figure 3 ). Furthermore, no statistically significant differences $(P>0.05)$ were detected between the cold and thermoneutral groups at any point in time ( 0 hours, 24 hours, 48 hours, and 72 hours) for VJP and PPO. Here, a large and significant effect over time was observed for $\operatorname{VJP}\left(F_{4,17}=32.541, P<0.001, \eta_{\text {Partial }}^{2}=0.884\right)$ and PPO $\left(F_{4,17}=32.541, P<0.001, \eta_{\text {Partial }}^{2}=0.884\right)$. Moreover, post hoc tests for time were statistically significant during the whole recovery period (0-72 hours) for VJP and PPO (Figure 3). However, there was no significant group $\times$ time 



Figure 2 Change over time of DOMS and RPE for the groups.

Notes: The values are in median \pm interquartile ranges. *Indicates a significant time effect $(P<0.05)$. \#Significantly different from baseline values within groups $(P<0.05)$. Abbreviations: DOMS, delayed-onset muscle soreness; RPE, ratings of perceived exertion; VAS, visual analog scale.

interaction for the VJP $\left(F_{4,17}=1.173, P=0.35, \eta_{\text {Partial }}^{2}=0.216\right)$ and the PPO groups $\left(F_{4,17}=1.173, P=0.35, \eta_{\text {Partial }}^{2}=0.216\right)$.

The individual $P$-values for the subjective and objective recovery variables are shown in Table 3 . The Pearsons' $r$ data analysis revealed strong negative inversed relationships in the cold group between the decrease in VJP and increase of DOMS ( $r=-0.88, P=0.018)$, as well as between decreased VJP and increased RPE ( $r=-0.87, P=0.022)$; these results were statistically significant. No significant correlations could be observed in the thermoneutral group between DOMS and VJP $(r=-0.76, P=0.07)$ or RPE and VJP $(r=-0.70, P=0.12)$.

\section{Discussion}

This study investigated the efficacy of a single cold application to enhance recovery following a $3 \times 30$ maximum jump task. The results clearly indicate that a single cold application failed to enhance the recovery process over a 72-hour recovery period. DOMS scores increased and VJP decreased during the 72-hour period following the jumping protocol.
This may be an indicator of provoked muscle damage, which can be seen in Figures 2 and 3. DOMS peaked after 48 hours in both groups which is consistent with the results of previous research. ${ }^{5,7}$ The objective measures, namely, VJP and the corresponding PPO, showed the greatest decline after 48 hours of recovery. The decline of the objective recovery variables might be related to the localized pain experienced in the participants' legs. The thermoneutral application led to a significant group effect over time for DOMS. This might indicate that the thermoneutral application was more beneficial than the cold application over time, but not at a specific time point. The perceived exertion peaked after 48 hours of recovery in the cold group but not in the thermoneutral group. Of particular note are the results for perceived exertion, which were lower between 48 hours and 72 hours of recovery in both groups (cold: $1.53 \%$, thermoneutral: $4.81 \%$ ). However, the thermoneutral group nearly reached the baseline values at the 72 hour follow-up measurement and the results ranged from borderline to significant between the two groups $(P=0.06)$. The RPE in the cold group was 


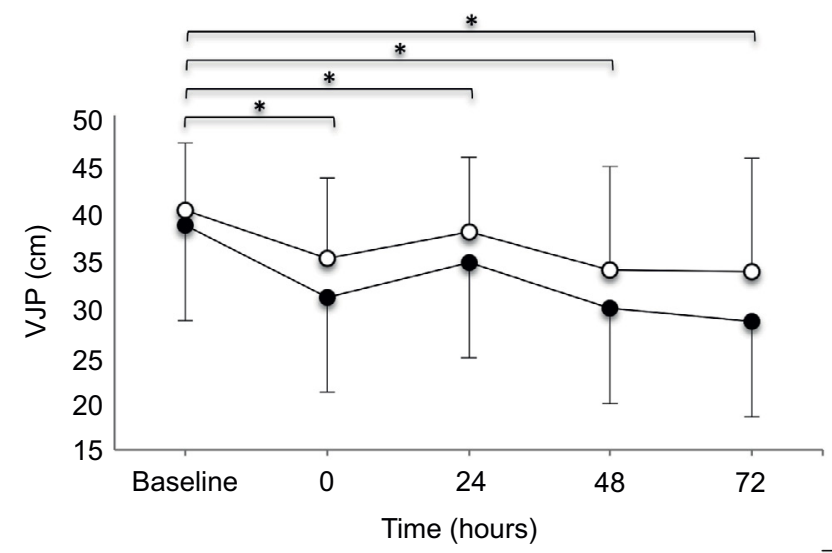

- Cold

- Thermoneutral

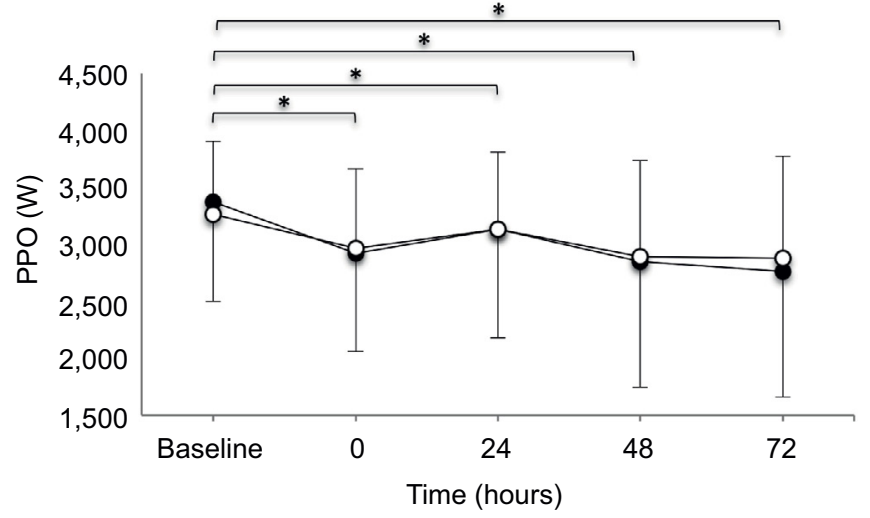

Figure 3 Change over time of VJP and PPO for the groups.

Notes: The values are provided as mean \pm SD. *Indicates a significant time effect $(P<0.05)$.

Abbreviations: PPO, peak power output; VJP, vertical jump performance; SD, standard deviation.

Table 3 Changes over time of subjective and objective recovery variables

\begin{tabular}{|c|c|c|c|c|c|}
\hline Variable & Baseline & 0 hours & 24 hours & 48 hours & 72 hours \\
\hline \multicolumn{6}{|c|}{ DOMS (VAS: 0-10 cm) } \\
\hline Cold group ${ }^{\mathrm{a}}$ & $0.1 \pm 0.0$ & $5 \pm 1.9$ & $5.6 \pm 2.2$ & $7.7 \pm 2.9$ & $7.7 \pm 3.75$ \\
\hline Thermoneutral group ${ }^{a}$ & $0.1 \pm 0.1$ & $4.1 \pm 3.35$ & $5.0 \pm 2.6$ & $6.3 \pm 3.2$ & $6.3 \pm 5.4$ \\
\hline$P$-value & 0.23 & 0.13 & 0.10 & 0.19 & 0.13 \\
\hline \multicolumn{6}{|l|}{ RPE (Borg: 6-20) } \\
\hline Cold group ${ }^{a}$ & $8 \pm 2$ & $14 \pm 2$ & $9 \pm 3.5$ & $12 \pm 5.5$ & $11 \pm 3.5$ \\
\hline Thermoneutral group ${ }^{a}$ & $7 \pm 2$ & $14 \pm 2$ & $8 \pm 2.5$ & $8 \pm 4.5$ & $7 \pm 4$ \\
\hline$P$-value & 0.20 & 1.00 & 0.24 & 0.11 & 0.06 \\
\hline \multicolumn{6}{|l|}{ VJP (cm) } \\
\hline Cold group & $38.5 \mathrm{I} \pm 6.86$ & $31.02 \pm 7.16$ & $34.59 \pm 8.07$ & $29.81 \pm 12.89$ & $28.44 \pm 13.77$ \\
\hline Thermoneutral group ${ }^{\mathrm{b}}$ & $40.08 \pm 6.99$ & $35.06 \pm 8.39$ & $37.82 \pm 7.76$ & $33.87 \pm 10.81$ & $33.65 \pm 11.92$ \\
\hline$P$-value & 0.60 & 0.23 & 0.35 & 0.43 & 0.35 \\
\hline \multicolumn{6}{|l|}{ PPO (W) } \\
\hline Cold group & $3,375 \pm 876$ & $2,920 \pm 855$ & $3,137 \pm 953$ & $2,846 \pm I, 105$ & $2,763 \pm 1,104$ \\
\hline Thermoneutral group ${ }^{\mathrm{b}}$ & $3,267 \pm 636$ & $2,963 \pm 702$ & $3,130 \pm 677$ & $2,890 \pm 844$ & $2,877 \pm 896$ \\
\hline$P$-value & 0.74 & 0.89 & 0.98 & 0.91 & 0.79 \\
\hline
\end{tabular}

Note: Values are presented as amedian \pm IQR and ${ }^{b}$ mean \pm SD.

Abbreviations: DOMS, delayed-onset muscle soreness; IQR, interquartile range; PPO, peak power output; RPE, ratings of perceived exertion; VAS, visual analog scale; VJP, vertical jump performance; SD, standard deviation. 
significantly higher after 72 hours compared to the baseline values $(P=0.002)$. The baseline $\mathrm{RPE}$ values were statistically not significantly different after 72 hours in the thermoneutral group $(P=0.10)$. This suggests that local cooling, immediately applied after an exhaustive jump protocol, could negatively affect well-being subjectively after 72 hours. The participants in the thermoneutral group reported feeling more comfortable during and after the thermoneutral application when compared to those in the cold group. A limitation of this study is that no muscle damage markers, such as C-reactive protein (CRP) or creatine-kinase (CK), were assessed. High-intensity exercise, unaccustomed activity, or exercise of long duration has been shown to induce inflammatory processes, with a rise in CRP. ${ }^{42,45}$ The degree of muscle damage is generally related to the amount of plasma CK by numerous researchers. ${ }^{28,46}$ These two factors are commonly used to describe the process of EIMD leading to DOMS. Although the reported objective parameters decreased and the subjective pain and RPE increased after the exhaustive jump protocol, it is impossible to say that real muscle damage occurred due to the absence of at least one of these biological markers. The target was for participants to obtain maximum muscle damage. After the exercise, all of the 22 participants had difficulties in managing their activities of daily living due to their pain symptoms (including climbing stairs and even walking). Neither the cold group nor the thermoneutral group returned to baseline values for DOMS following the 72-hour recovery period. The authors are aware that the thermotherapy might have had a more positive influence on recovery if the impact of the exhaustive exercise had been moderate. It is documented that the amount of adipose tissue is a significant factor in the extent of intramuscular temperature change during and after cryotherapy ${ }^{47-49}$ In this study, the participants had very similar body mass indexes and, of particular interest, an estimated low body fat percentage (Table 1), minimizing the risk of adipose tissue-influenced temperature changes. However, additional skinfold measurements would have been useful for further clarity. There is controversy in current literature regarding the effect of cooling on muscle tissue. Available evidence indicates that cryotherapy or icing, as currently practiced, is unlikely to be successful in cooling human muscle tissue sufficiently because the temperature reductions in humans are only moderate in comparison to that in the most commonly used animal models. ${ }^{50}$ Recently published results indicated that thermoneutral placebo water immersion was superior in the recovery of muscle strength over 48 hours, compared to a thermoneutral water immersion, and is also just as effective as CWI.${ }^{51}$ The authors concluded that this result could be attributed to improved subjective ratings, suggesting that the commonly hypothesized physiological benefits surrounding cryotherapy could be explained, in part by the placebo effect. More studies investigating the physiological effects of cryotherapy on human recovery might help to understand these mechanisms.

\section{Conclusion}

Thus, local cryotherapy had no significant influence on local pain ratings (DOMS) during or after 72 hours of recovery. The thermoneutral group showed a trend toward alleviating the symptoms of perceived exertion after 72 hours. This may provide a chance for these participants for a psychological advantage during their recovery. The thermoneutral group returned to baseline values for RPE after 72 hours, which was not observed in the cold group.

The cold application also failed to positively influence the objective recovery variables. However, local cooling seems to have no detrimental effect on recovery. In this experimental study, a local cryotherapy cuff application for the duration of 20 minutes failed to positively affect recovery characteristics.

\section{Acknowledgments}

The authors thank the "Thim van der Laan" Foundation, Landquart, Switzerland, for their financial support. We also thank Thomas Konzett, University of Applied Sciences and Arts of Southern Switzerland, Landquart, Switzerland, and Ursula M Küng, University College Physiotherapy, Landquart, Switzerland, for their assistance during the experiment, as well as Lindsay Kunz-Melcher for her assistance with English.

\section{Disclosure}

The authors report no conflicts of interest in this work.

\section{References}

1. Hohenauer E, Taeymans J, Baeyens JP, Clarys P, Clijsen R. The effect of post-exercise cryotherapy on recovery characteristics: a systematic review and meta-analysis. PLoS One. 2015;10(9):e0139028.

2. Costello JT, Baker PR, Minett GM, Bieuzen F, Stewart IB, Bleakley C. Whole-body cryotherapy (extreme cold air exposure) for preventing and treating muscle soreness after exercise in adults. Cochrane Database Syst Rev. 2015;9:CD010789.

3. White GE, Wells GD. Cold-water immersion and other forms of cryotherapy: physiological changes potentially affecting recovery from high-intensity exercise. Extrem Physiol Med. 2013;2(1):26.

4. Mawhinney C, Jones H, Joo CH, Low DA, Green DJ, Gregson W. Influence of cold-water immersion on limb and cutaneous blood flow after exercise. Med Sci Sports Exerc. 2013;45(12):2277-2285.

5. Tseng CY, Lee JP, Tsai YS, et al. Topical cooling (icing) delays recovery from eccentric exercise-induced muscle damage. J Strength Cond Res. 2013;27(5):1354-1361. 
6. Pointon M, Duffield R, Cannon J, Marino FE. Cold application for neuromuscular recovery following intense lower-body exercise. Eur $J$ Appl Physiol. 2011;111(12):2977-2986.

7. Guilhem G, Hug F, Couturier A, et al. Effects of air-pulsed cryotherapy on neuromuscular recovery subsequent to exercise-induced muscle damage. Am J Sports Med. 2013;41(8):1942-1951.

8. Leicht AS, Sinclair WH, Patterson MJ, et al. Influence of postexercise cooling techniques on heart rate variability in men. Exp Physiol. 2009;94(6):695-703.

9. Ingram J, Dawson B, Goodman C, Wallman K, Beilby J. Effect of water immersion methods on post-exercise recovery from simulated team sport exercise. J Sci Med Sport. 2009;12(3):417-421.

10. Hausswirth C, Louis J, Bieuzen F, et al. Effects of whole-body cryotherapy vs. far-infrared vs. passive modalities on recovery from exercise-induced muscle damage in highly-trained runners. PLoS One. 2011;6(12):e27749.

11. Ferreira-Junior JB, Bottaro M, Vieira CA, et al. Effects of partial-body cryotherapy $\left(-110^{\circ} \mathrm{C}\right)$ on muscle recovery between high-intensity exercise bouts. Int J Sports Med. 2014;35(14):1155-1160.

12. Minett GM, Duffield R, Kellett A, Portus M. Effects of mixed-method cooling on recovery of medium-fast bowling performance in hot conditions on consecutive days. J Sports Sci. 2012;30(13):1387-1396.

13. Stanley J, Leveritt M, Peake JM. Thermoregulatory responses to iceslush beverage ingestion and exercise in the heat. Eur J Appl Physiol. 2010;110(6):1163-1173.

14. Tran Trong T, Riera F, Rinaldi K, Briki W, Hue O. Ingestion of a cold temperature/menthol beverage increases outdoor exercise performance in a hot, humid environment. PLoS One. 2015;10(4):e0123815.

15. Siegel R, Mate J, Watson G, Nosaka K, Laursen PB. Pre-cooling with ice slurry ingestion leads to similar run times to exhaustion in the heat as cold water immersion. $J$ Sports Sci. 2012;30(2):155-165.

16. Hue O, Monjo R, Lazzaro M, et al. The effect of time of day on cold water ingestion by high-level swimmers in a tropical climate. Int $J$ Sports Physiol Perform. 2013;8(4):442-451.

17. King M, Duffield R. The effects of recovery interventions on consecutive days of intermittent sprint exercise. $J$ Strength Cond Res. 2009; 23(6):1795-1802.

18. Delextrat A, Calleja-Gonzalez J, Hippocrate A, Clarke ND. Effects of sports massage and intermittent cold-water immersion on recovery from matches by basketball players. J Sports Sci. 2013;31(1):11-19.

19. Stanley J, Peake JM, Buchheit M. Consecutive days of cold water immersion: effects on cycling performance and heart rate variability. Eur J Appl Physiol. 2013;113(2):371-384.

20. Rowsell GJ, Coutts AJ, Reaburn P, Hill-Haas S. Effects of cold-water immersion on physical performance between successive matches in high-performance junior male soccer players. J Sports Sci. 2009; 27(6):565-573.

21. Cheung SS, Sleivert GG. Lowering of skin temperature decreases isokinetic maximal force production independent of core temperature. Eur J Appl Physiol. 2004;91(5-6):723-728.

22. Bleakley C, McDonough S, Gardner E, Baxter GD, Hopkins JT, Davison GW. Cold-water immersion (cryotherapy) for preventing and treating muscle soreness after exercise. Cochrane Database Syst Rev. 2012;2:CD008262.

23. Cheung K, Hume P, Maxwell L. Delayed onset muscle soreness: treatment strategies and performance factors. Sports Med. 2003;33(2):145-164.

24. Costello JT, Algar LA, Donnelly AE. Effects of whole-body cryotherapy $\left(-110^{\circ} \mathrm{C}\right)$ on proprioception and indices of muscle damage. Scand J Med Sci Sports. 2012;22(2):190-198.

25. Crowe MJ, O'Connor D, Rudd D. Cold water recovery reduces anaerobic performance. Int J Sports Med. 2007;28(12):994-998.

26. Vieira A, Bottaro M, Ferreira-Junior JB, et al. Does whole-body cryotherapy improve vertical jump recovery following a high-intensity exercise bout? Open Access J Sports Med. 2015;6:49-54.

27. Borg GA. Psychophysical bases of perceived exertion. Med Sci Sports Exerc. 1982;14(5):377-381.

28. Goodall S, Howatson $\mathrm{G}$. The effects of multiple cold water immersions on indices of muscle damage. $J$ Sports Sci Med. 2008;7(2):235-241.
29. Rupp KA, Selkow NM, Parente WR, Ingersoll CD, Weltman AL, Saliba SA. The effect of cold water immersion on 48-hour performance testing in collegiate soccer players. J Strength Cond Res. 2012;26(8):2043-2050.

30. Sayers SP, Harackiewicz DV, Harman EA, Frykman PN, Rosenstein MT. Cross-validation of three jump power equations. Med Sci Sports Exerc. 1999;31(4):572-577.

31. McMahon JJ, Jones PA, Comfort P. A correction equation for jump height measured using the just jump system. Int $J$ Sports Physiol Perform. 2016;11(4):555-557.

32. Markovic G, Dizdar D, Jukic I, Cardinale M. Reliability and factorial validity of squat and countermovement jump tests. J Strength Cond Res. 2004;18(3):551-555.

33. Slinde F, Suber C, Suber L, Edwen CE, Svantesson U. Test-retest reliability of three different countermovement jumping tests. J Strength Cond Res. 2008;22(2):640-644.

34. Ferreira-Junior JB, Bottaro M, Vieira A, et al. One session of partialbody cryotherapy (-110 degrees C) improves muscle damage recovery. Scand J Med Sci Sports. 2015;25(5):e524-e530.

35. Twist C, Eston R. The effects of exercise-induced muscle damage on maximal intensity intermittent exercise performance. Eur J Appl Physiol. 2005;94(5-6):652-658.

36. Versey NG, Halson SL, Dawson BT. Water immersion recovery for athletes: effect on exercise performance and practical recommendations. Sports Med. 2013;43(11):1101-1130.

37. Takahashi J, Ishihara K, Aoki J. Effect of aqua exercise on recovery of lower limb muscles after downhill running. J Sports Sci. 2006;24(8):835-842.

38. Sramek P, Simeckova M, Jansky L, Savlikova J, Vybiral S. Human physiological responses to immersion into water of different temperatures. Eur J Appl Physiol. 2000;81(5):436-442.

39. Nishimura M, Onodera S. Effects of supine floating on heart rate, blood pressure and cardiac autonomic nervous system activity. $J$ Gravit Physiol. 2000;7(2):171-172.

40. Sellwood KL, Brukner P, Williams D, Nicol A, Hinman R. Ice-water immersion and delayed-onset muscle soreness: a randomised controlled trial. Br J Sports Med. 2007;41(6):392-397.

41. Rowsell GJ, Coutts AJ, Reaburn P, Hill-Haas S. Effect of post-match cold-water immersion on subsequent match running performance in junior soccer players during tournament play. J Sports Sci. 2011;29(1): $1-6$.

42. Ascensao A, Leite M, Rebelo AN, Magalhaes S, Magalhaes J. Effects of cold water immersion on the recovery of physical performance and muscle damage following a one-off soccer match. J Sports Sci. 2011; 29(3):217-225

43. Dawson B, Cow S, Modra S, Bishop D, Stewart G. Effects of immediate post-game recovery procedures on muscle soreness, power and flexiblity levels over the next 48 hours. J Sci Med Sport. 2005;8(2):210-221.

44. Cohen J. A power primer. Psychol Bull. 1992;112(1):155-159.

45. Pointon M, Duffield R, Cannon J, Marino FE. Cold water immersion recovery following intermittent-sprint exercise in the heat. Eur J Appl Physiol. 2012;112(7):2483-2494.

46. Bailey DM, Erith SJ, Griffin PJ, et al. Influence of cold-water immersion on indices of muscle damage following prolonged intermittent shuttle running. J Sports Sci. 2007;25(11):1163-1170.

47. Myrer WJ, Myrer KA, Measom GJ, Fellingham GW, Evers SL. Muscle temperature is affected by overlying adipose when cryotherapy is administered. J Athl Train. 2001;36(1):32-36.

48. Otte JW, Merrick MA, Ingersoll CD, Cordova ML. Subcutaneous adipose tissue thickness alters cooling time during cryotherapy. Arch Phys Med Rehabil. 2002;83(11):1501-1505.

49. Jutte LS, Merrick MA, Ingersoll CD, Edwards JE. The relationship between intramuscular temperature, skin temperature, and adipose thickness during cryotherapy and rewarming. Arch Phys Med Rehabil. 2001;82(6):845-850.

50. Bleakley CM, Glasgow P, Webb MJ. Cooling an acute muscle injury: can basic scientific theory translate into the clinical setting? Br J Sports Med. 2012;46(4):296-298.

51. Broatch JR, Petersen A, Bishop DJ. Postexercise cold water immersion benefits are not greater than the placebo effect. Med Sci Sports Exerc. 2014;46(11):2139-2147. 
The Open Access Journal of Sports Medicine is an international, peer-reviewed, open access journal publishing original research, reports, reviews and commentaries on all areas of sports medicine. The journal is included on PubMed. The manuscript management system is completely online and includes a very quick and fair peer-review system. Visit http://www.dovepress.com/testimonials.php to read real quotes from published authors.

Submit your manuscript here: http://www.dovepress.com/open-access-journal-of-sports-medicine-journal 